loop. This has to be sterilised in a flame before each use. It upsets many patients if they see the flaming, as they are liable to do if it is done on the ward. Moreover, the metal tends to cut into the solid medium of the plates inoculated, which renders isolation of any growth difficult.

A highly acceptable improvement is the presterilised plastic disposable bacteriological loop. In South Glamorgan 10- $\mu$ l capacity loops (made by Nunc Ltd.) are bought in bulk, packed singly, and sterilised in $15 \%$ ethylene oxide in carbon dioxide mixture for three hours in the central sterile services department. These loops easily enter the urethra and do not traumatise patient or bacteriological medium. Vaginal wet films are easily taken for direct microscopic examination for Trichomonas vaginalis.

Decreasing the difficulties in specimentaking increases the motivation of the medical staff to perform adequate investigation and may be one factor in the $29 \%$ isolation rate for Neisseria gonorrhoeae in salpingitis patients in this area.

R A SPARKS A J Davies

St David's Hospital,
Cardiff

Sparks, R A, and Davies, A J, British fournal of

Venereal Diseases. In press.

\section{Otitis media}

SIR,-I disagree with the opinion expressed by $\mathrm{Mr} \mathrm{J} \mathrm{F}$ Birell in his original article (21 February, p 443) and subsequent letter (3 April, p 836)

I have on numerous occasions seen children complaining of severe unilateral earache with a temperature and signs of an upper respiratory infection in whom there was soft, beige-yellow wax in the affected ear. On the occasions I have removed this wax-either by syringing or with a probe-an inflamed red drum behind the wax has been revealed. I no longer do this, principally because it is too painful in such a situation. I regard the presence of such wax as diagnostic and therefore needing appropriate antibiotic therapy.

Occasionally the mother will say there has been a discharge from the ear, sometimes describing it as "bloody." I have assumed that the temperature of the inflamed middle ear has melted the wax, causing some of it to run and subsequently the colour to change.

STUART CARNE

London W12

SIR,-In his article $\mathrm{Mr}$ J F Birrell (21 February, $p$ 443) did not mention myringotomy in the treatment of acute otitis media. However, surgical drainage is one of the basic principles in the treatment of empyema. In otitis media the functioning of the congested Eustachian tube and the clearing activity of the middle ear epithelial cilia are impaired and this occasions the accumulation and stagnation of inflammatory exudate in the middle ear-a situation resembling empyema. The accumulated exudate can then corrode its way through the drum causing necrotic perforation, which takes much longer to heal than a small myringotomy hole made through a "healthy" drum. Thus early paracentesis can in many cases prevent spontaneous necrotic perfora- tion and its consequences. On the other hand stagnation of exudate in the middle ea lengthens the course of infection. Friedmann, in his experimental studies, found proliferation of mucous elements and formation of glands in the middle ear mucosa even after only two weeks of inflammatory process. Thus a prolonged course of infection probably plays an important role in the development of secretory otitis media, a process that is characterised by the proliferation of the secretory elements of the middle ear mucosa Our finding ${ }^{2}$ of the dominance of lymphocyte and neutrophils in smears made of 137 glueear secretions and the finding of bacteria in one-third of those smears further support the infectious aetiology of the disease. Consequently I consider the performance of myringotomy to be of the utmost importance in bringing about environmental condition that are as advantageous as possible for the resolution of the infection.

Fluid in the middle ear impedes mobility of the drum, the appearance of which can sometimes be misleading. With the aid of a pneumatic speculum the movements of the drum can be visualized. If fluid is suspected myringotomy under local or general anesthesia must be performed, the incision of the drum always being followed by aspiration of the fluid from the middle ear.

As $\mathrm{Mr}$ Birrell states, pain is the principal symptom in acute otitis media. Myringotomy gives immediate relief from pain. Since, at the same time, myringotomy provides reliable information on the nature of the middle ear process and also creates as favourable conditions for rapid healing as are possible under the circumstances there seems no justification for not using this widely advocated ${ }^{3}$ procedure.

Department of Otolaryngology

P KARMA University of Oulu Friedmann, I, fournal of Laryngology and Otology,
1955, 69, 588. Palva, T, Holopainen, E, and Karma, P, Annals of Otology, Rhinology and Laryngology. In press.

Taylor, L, in Scott-Brown's Diseases of the Ear, Nose and Throat, ed J Ballantyne and J Groves, vol 2,

\section{Psychosurgery on television}

SIR,-This unit has more experience of contemporary stereotactic psychosurgical operations (and we do not refer to the obsolete earlier leucotomies) than any other department in Britain and probably in the world.

On the basis of this experience we were most disturbed to see a programme on Independent Television on 30 March. This showed a patient who had a psychosurgical operation apparently because of abnormally aggressive behaviour. The indications for psychosurgery in this case would have proved controversial in any medical conference and many would have grave doubts about the type of operation performed. It seems to us, therefore, to be quite irresponsible to present, and in some lurid detail, such an extremely unusual clinical problem for popular consumption.

It is generally realised that the use of psychosurgery for abnormally aggressive behaviour presents especially difficult ethical and clinical problems and such operations are very rare in Britain. As a result of the television programme, which lasted an hour, the public will confuse the controversial case shown to them with the increasingly accepted type of psychosurgery carried out both at this unit and at the Atkinson Morley Hospital for certain severe psychiatric illnesses which have been described by us in several publications. ${ }^{1-3}$

Is it not possible for some kind of control to be exerted on the mass media or are we to allow their presentations to be solely dictated by a need for the dramatic and the extreme?

P K BRIDGES JOHN R BARTLETT

Geoffrey Knight Psychosurgical Unit,

Brook General Hospital, SE18

Bridges, P K, and Bartlett, J R, Postgraduate Medical Fournal, 1973, 49, 855

Goktepe, E O, Young, L B, and Bridges, P K, British fournal of Psychiatry, 1975, 126, 270.

Psychiatry, 1971, 118, 141 .

\section{An eye-pad hazard}

SIR,-While the obvious lesson to be derived from Dr R Wall's account of inflammable eye pads (27 March, p 772) is that they should be made non-inflammable, might one also stress the equally important but less obvious lessonwas this and indeed any other eye pad strictly necessary?

For a long while it has become increasingly clear that in very many cases the traditional use of eye pads - for example, after outpatient procedures, the incision of meibomian cysts, and even tonometries-is not only totally unnecessary and a source of considerable discomfort to patients but may additionally be actively harmful as the cause of pad abrasions. It is to be hoped that the non-ophthalmological doctor and nurse will take due notice of the very marked changes that have taken place in accepted and advised practice in this matter.

M J Gilkes

Sussex Eye Hospital,

Brighton

SIR,-My recent letter concerning the inflammability of eye pads (27 March, p 772) contained a reference to John Dickinson and Co Ltd. I have since been informed that this company is responsible only for the packaging of the eye pads in question, which they neither manufacture nor supply. I wish to apologise for any embarrassment that they have suffered.

R WALL

Highcroft Hospital,

Birmingham

\section{Specialties within community medicine}

SIR,-As a trainee in community medicine I write to endorse Dr A S St Leger's views (20 March, $p$ 709). We are being trained rather than educated and the syllabus embodies many of the epistemological mistakes that have hindered progress for so long. Sociology and administrative theory are full of untestable hypotheses and, as Dr St Leger says, our study of the other topics is so limited that the worth in them is not fully appreciated.

It is obvious that community medicine is becoming committee medicine and that community physicians are dealing with Health 